\title{
Validation of a Decision Rule and Derivation of a Modified Rule to Obtain Chest Radiograph in Patients with Nontraumatic Chest Pain in the Emergency Department
}

\author{
Case Newsom, ${ }^{1}$ Rebecca Jeanmonod, ${ }^{1}$ Karl Weller, ${ }^{1}$ Nabil Boutros, ${ }^{2}$ \\ Mark Reiter, ${ }^{2}$ and Donald Jeanmonod ${ }^{1}$ \\ ${ }^{1}$ St. Luke's University Hospital and Health Network, Department of Emergency Medicine, Bethlehem, PA 610-954-1102, USA \\ ${ }^{2}$ University of Tennessee School of Medicine, Middle Tennessee Emergency Physicians, PC, 1 Vantage Way No. B240, \\ Nashville, TN 37228-1562, USA \\ Correspondence should be addressed to Rebecca Jeanmonod; rebeccajeanmonod@yahoo.com
}

Received 2 March 2014; Revised 19 May 2014; Accepted 27 May 2014; Published 12 June 2014

Academic Editor: Bruno Megarbane

Copyright @ 2014 Case Newsom et al. This is an open access article distributed under the Creative Commons Attribution License, which permits unrestricted use, distribution, and reproduction in any medium, provided the original work is properly cited.

\begin{abstract}
Objectives. We sought to validate and refine a decision rule for chest X-ray (CXR) utilization in nontraumatic chest pain (CP) patients presenting to the emergency department (ED). Methods. Retrospective review of ED patients presenting with CP who had CXR performed during three nonconsecutive months was performed. The presence of 18 variables derived from history and exam was ascertained. Sensitivity, specificity, positive predictive value (PPV), and negative predictive value (NPV) of the original rule were calculated. Refinement using additional variables was performed. Results. 967 patient charts were reviewed. 89.9\% of CXR were normal, 5.2\% had insignificant findings, and 5.1\% had significant findings. Application of the criteria had a sensitivity/specificity of $74 \% / 59 \%$ and a PPV/ NPV of $9 \% / 98 \%$. Rule modification to obtain CXR for age $\geq 65$ years, history of congestive heart failure and alcohol abuse, and exam findings of decreased breath sounds, fever, and tachypnea maintained sensitivity while improving specificity to $69 \%$. Conclusions. Most CP patients have normal CXRs. Narrowing a decision rule to obtain CXR in patients with age $\geq 65$ years, history of congestive heart failure and alcohol abuse, and exam findings of decreased breath sounds, fever, and tachypnea maintain sensitivity while improving specificity and NPV.
\end{abstract}

\section{Introduction}

Chest pain remains one of the most common patient presentations to the emergency department (ED). In fact, a study from 2005 demonstrated more than 6 million annual ED visits with a chief complaint of chest pain [1]. The differential diagnosis of chest pain is extensive, and a handful of immediately life-threatening causes must be considered and excluded when evaluating a patient presenting with chest pain. While the utility of EKG and troponin testing in the evaluation of patients with potential acute coronary syndrome has been demonstrated $[2,3]$, there is a paucity of evidence to support the routine use of chest radiography. Additionally, lack of certain signs and symptoms may obviate the need for chest radiography in the evaluation for other concerning causes of chest pain. Chest radiographs are very useful at times but are found to be unremarkable the vast majority of the time. A clinical decision rule (CDR) for the use of chest radiography in patients presenting with nontraumatic chest pain could reduce the use of chest radiography, enhance ED throughput, decrease costs, and decrease radiation exposure. Several recent studies have attempted to identify the signs and symptoms associated with abnormal and actionable findings on chest radiographs. In 2002, a CDR was developed using retrospective data by Rothrock et al. [4], which included patients with any nontraumatic complaints referable to the chest (e.g., shortness of breath or chest pain). In this study, the overall incidence of clinically significant radiographic findings in the population was $17 \%$, with the CDR showing a 98\% (95\% CI 96-99\%) negative predictive 
value if none of the following criteria were met: age $\geq 60$ years, temperature $\geq 100.4^{\circ} \mathrm{F}\left(38^{\circ} \mathrm{C}\right)$, oxygen saturation $<90 \%$, respiratory rate $>24$ breaths/min, hemoptysis, rales, diminished breath sounds, a history of alcohol abuse, tuberculosis, or thromboembolic disease [4]. The positive predictive value of any of the above criteria was $25 \%$ (95\% CI 23-27\%) and the study has yet to be prospectively validated.

In 2010, a study on patients with presentations concerning symptomatic coronary disease found that those with no history of congestive heart failure, no history of smoking, and no abnormalities on lung auscultation could forgo chest radiography [5]. In this study, the overall incidence of positive findings on chest radiography was $2.1 \%$ [5]. The CDR was $100 \%$ sensitive but failed a prospective validation in 2012 [6]. This resulted in a refined CDR, which included the following criteria: no shortness of breath, no history of smoking, no abnormalities on lung auscultation, and age $<55$. While these criteria achieved a negative predictive value of 100\% (95\% CI 96.3-100\%), the prediction rule lacked specificity, with a reported specificity of $11.5 \%$ (95\% CI 9.6-13.5\%) [6].

We sought to assess which variables that were found to be significantly predictive in prior studies are associated with clinically relevant abnormal findings. We further attempted to refine a CDR to guide selective chest radiography in the patient with nontraumatic chest pain.

\section{Materials and Methods}

2.1. Study Design. This study examined a retrospective cohort of patients presenting to the emergency department with a chief complaint of chest pain. The research protocol was reviewed and approved by the institutional review board at the study facility. Because of the retrospective design, written informed consent was waived.

2.2. Study Setting and Population. The study site is a level 1 community trauma center with an ED census of 65,000 that hosts an emergency medicine residency program. The study site uses McKesson patient tracker software and Horizon HP Physician Portal electronic medical record (McKesson Corporation, 2011). The electronic ED record was queried for all adult patients triaged by nursing with a chief complaint of "chest pain" during three nonconsecutive months in 2011 (January, April, and October) to best represent the seasonal variability of abnormal chest radiography. Only patients that underwent chest radiography were included in review. Patients were not excluded on the basis of additional chief complaints (e.g., chest pain and shortness of breath) nor were they excluded on the basis of abnormal vital signs. Patients were excluded if they were triaged as trauma patients to the trauma bay.

2.3. Study Protocol and Measurements. Chest radiography was performed at the discretion of the treating physician or as part of a protocolized order set for patients presenting with chest pain. Radiography results were recorded in the standardized spreadsheet. For each patient visit, the ED chart was systematically reviewed for the presence of 18 historical and physical exam variables of interest. These variables were chosen based on prior literature that suggested they may be associated with abnormal findings on chest radiography. The variables were recorded from a closed list of possibilities in a standardized Excel spreadsheet by 4 trained research associates. These variables included the patient age, history of hemoptysis, thromboembolic disease (prior history of deep venous thrombosis or pulmonary embolus), alcohol abuse, tuberculosis, asthma, chronic obstructive pulmonary disease, coronary artery disease (history of coronary artery disease listed on the chart or history of myocardial infarction), congestive heart failure, history of tuberculosis, and examination findings of rales, rhonchi, decreased breath sounds, wheezes, chest tenderness, lower extremity edema, temperature greater than $100.4^{\circ} \mathrm{F}$, oxygen saturation less than $90 \%$, and respiratory rate greater than 24 . Only vital signs from initial presentation were used. The historical variables were assessed as being present if they were listed in the nursing, attending physician, or resident documentation. The physical exam variables were assessed as being present if they were recorded in either the resident or the attending physician documentation. If a variable was not mentioned in the record, it was assessed as not being present. No attempt was made to review prior or subsequent charts to look for missing historical data. Given that all patients reviewed had chest radiographs performed, radiography results were recorded in the standardized spreadsheet. Staff radiologist interpretations were used as the gold standard for defining all chest radiograph abnormalities. In cases where the staff radiologist read did not provide a definitive diagnosis (e.g., "pneumonia versus atelectasis"), the ED diagnosis was used as the gold standard. Abnormal radiographs were stratified a priori into clinically significant and clinically insignificant acute findings. Clinically significant findings included pneumonia, pleural effusion, pneumothorax, congestive heart failure, or the presence of a new mass. In addition to recording the presence or absence of study variables, the patient's disposition (admission or discharge), ED diagnosis, and inpatient diagnoses were recorded.

2.4. Data Analysis. Using a presumed incidence of clinically significant chest radiograph findings of $17 \%$ (as per Rothrock's study [4]) and a target of 10 positive radiographs for each variable analyzed, we sought to enroll 1000 patients and anticipated that 3 months of patient encounters for "chest pain" would be sufficient to meet this goal. Variables corresponding to Rothrock's CDR were analyzed for specificity and sensitivity to validate this rule. The variable of "history of tuberculosis" was excluded because of the extremely low incidence of this historical feature in our population. The 18 variables in total were analyzed using both direct and stepwise logistic regression to determine the best prediction rule for clinically relevant abnormal chest radiography, and odds ratios and $P$ values were calculated. General demographic data, patient disposition, and diagnostic information were collected and analyzed with descriptive statistics. Data were analyzed using MedCalc (1993-2013, Ostend, Belgium). 
TABLE 1: Final diagnoses of patients assigned by emergency physician and admitting physician.

\begin{tabular}{lcc}
\hline & ED assigned final diagnosis at disposition & Admitted patients final diagnosis at discharge \\
Final diagnosis & $N=967$ & $N=53$ \\
& $N(\%)$ & $203(34.8)$ \\
\hline Nonspecific "chest pain" & $589(60.9)$ & $93(16.0)$ \\
CAD related (MI, ACS, angina, etc.) & $80(8.3)$ & $64(11)$ \\
Musculoskeletal chest pain & $67(6.9)$ & $14(2.4)$ \\
CHF & $18(1.9)$ & $13(2.2)$ \\
Pneumonia & $15(1.6)$ & $42(7.2)$ \\
Gastritis/GERD & $15(1.6)$ & $10(1.7)$ \\
Pulmonary embolism & & \\
\hline
\end{tabular}

TABLE 2: Clinically insignificant abnormal findings on chest radiography.

\begin{tabular}{lc}
\hline Radiographic abnormality & $\begin{array}{c}\text { Number of patients with } \\
\text { finding (\% of total) }\end{array}$ \\
\hline Atelectasis & $19(2.0 \%)$ \\
Cardiomegaly & $13(1.3 \%)$ \\
Nodule & $4(0.4 \%)$ \\
Chronic obstructive pulmonary disease & $2(0.2 \%)$ \\
Hiatal hernia & $2(0.2 \%)$ \\
Known mass & $2(0.2 \%)$ \\
Other & $9(0.9 \%)$ \\
\hline
\end{tabular}

\section{Results}

967 patient charts were reviewed. The mean age of patients was 52 (interquartile range $40-67) .39 .7 \%$ of patients $(n=$ $384)$ were discharged home and $60.3 \%(n=583)$ were admitted to the hospital, some for treatment of the chest radiograph findings but mostly for serial cardiac enzyme assessment and any other workup unrelated to negative or insignificant chest radiographs. While most patients, both those in the ED and those admitted to the hospital, were diagnosed with nonspecified "chest pain," there were a significant number of specific causes of nontraumatic chest pain diagnosed (Table 1).

$89.6 \%$ of patients had a chest radiograph that was read as either "normal" or "no acute abnormality." 51 patients (5.3\%) had radiographic abnormalities that were clinically insignificant. The radiographic diagnoses for these patients are listed in Table 2.50 patients (5.2\%) had chest radiography abnormalities that were clinically significant. These diagnoses are graphically represented in Figure 1. On review of these significant diagnoses and the related ED chart, 3 of these were determined to be traumatic injuries in patients presenting related to blunt trauma to the chest. These cases were excluded from further analysis. There were no other patients enrolled in the study who were under evaluation for trauma.

The historical and physical exam elements that were associated with clinically significant abnormal findings on chest radiography were age, history of alcohol abuse, history of congestive heart failure, decreased breath sounds, fever, hypoxia, and tachypnea, respectively (Table 3). When applied
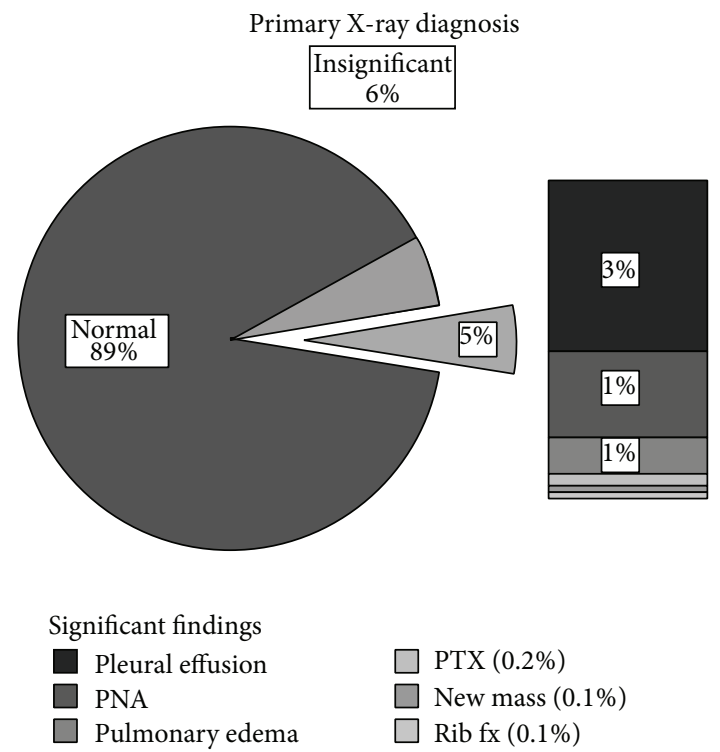

FIgURE 1: Primary X-ray diagnosis as read by staff radiologists.

to our patients, Rothrock's high yield criteria (oxygen saturation $<90 \%$, age $\geq 60$, decreased breath sounds, presence of rales, respiratory rate $>24$, history of thromboembolic disease, history of alcohol abuse, history of tuberculosis, hemoptysis, or presence of temperature $\geq 38^{\circ} \mathrm{C}$ ) had a sensitivity of $79 \%$ (95\% CI 64-89\%), a specificity of 59\% (95\% CI 55-62\%), a positive predictive value of $9 \%$ (95\% CI 6-12\%), and a negative predictive value of $98 \%$ (95\% CI 97-99\%). Modifying these criteria to include an older age group $(\geq 65)$, to add history of CHF, to remove history of thromboembolic disease, hemoptysis, or tuberculosis, and to remove exam findings of decreased oxygen saturation and the presence of rales preserved the sensitivity and negative predictive value of the rule and increased its specificity to $69 \%$ (95\% CI 66$72 \%)$, with a positive predictive value of 11 (95\% CI $8-16 \%)$. For the 10 patients who were decision rule negative but had significant findings on chest radiography, 5 had radiographic pneumonia, 3 had pleural effusion, 1 had pulmonary edema, and 1 had a new mass. Both the original rule and the modified rule failed to identify these 10 patients. 
TABLE 3: Associations of historical and physical findings with clinically relevant radiographic abnormalities on chest radiography.

\begin{tabular}{lcc}
\hline Variable & Odds ratio $(\mathrm{CI})$ & $P$ value \\
\hline Age $\geq 65$ & $3.3(1.6-6.9)$ & 0.001 \\
History of coronary disease & $0.69(0.31-1.6)$ & 0.37 \\
History of asthma & $0.56(0.15-2.1)$ & 0.39 \\
History of COPD & $0.66(0.18-2.4)$ & 0.54 \\
History of CHF & $9.4(3.7-24)$ & $<0.0001$ \\
History of VTE & $0.98(0.21-4.5)$ & 0.98 \\
History of alcohol abuse & $9.5(2.2-41)$ & 0.003 \\
Hemoptysis & $2.6(0.10-68)$ & 0.56 \\
Fever & $7.5(1.8-30)$ & 0.005 \\
Hypoxia & $9.6(1.0-90)$ & 0.05 \\
Tachypnea & $4.2(1.3-14)$ & 0.02 \\
Wheezing & $2.7(0.66-11)$ & 0.17 \\
Rales & $2.0(0.39-9.7)$ & 0.41 \\
Rhonchi & $2.8(0.42-19)$ & 0.29 \\
Decreased breath sounds & $5.1(1.2-23)$ \\
Tender chest wall & $2.4(0.86-6.8)$ & 0.03 \\
Swollen lower extremity & $1.6(0.61-4.3)$ & 0.09 \\
\hline
\end{tabular}

\section{Discussion}

As concern for radiation risk rises in the collective medical consciousness simultaneously with interest in medical cost containment, emergency providers are seeking more rational approaches for ordering diagnostic studies. The ubiquitous ordering of chest radiographs in patients with atraumatic chest pain makes an obvious target for further evaluation for clinical utility.

In a prior prospective study with a sample size of about 300 patients, $23 \%$ of patients had an abnormal chest radiograph influencing the patient's management [7]. This suggests that chest radiography is indispensable in the emergency evaluation of chest pain, as its findings alter management decisions. In contrast to this, Hess's study found significant abnormal chest radiographs in only about $2 \%$ of patients enrolled [5].

In our population, the incidence of clinically significant positive chest radiograph findings for patients presenting to the ED with nontraumatic chest pain was only $5 \%$. Of these, $80 \%$ were identified using the modified Rothrock criteria (age over 65, history of alcohol use, history of congestive heart failure, fever, hypoxia, tachypnea, or decreased breath sounds). In a population such as ours with a low incidence of disease, the negative predictive value using these criteria is $98 \%$, with $2 \%$ of patients having missed pathology.

In the $1 \%$ of all patients who would have had a missed pneumonia, this miss may be clinically irrelevant. While many pneumonias are dangerous if missed, it is another area of study to render risk ratios for poor outcomes of missed pneumonias as a whole. Many pneumonias are viral or more benign atypical pneumonias and, in our sample population of triaged "chest pain" patients, only one patient with pneumonia was admitted to the hospital and that patient had a concomitant diagnosis of pericarditis. The subset of patients with pneumonia missed by the rule had a mean age of
36 years. Although early antibiotics is a tenet of management of all bacterial infectious processes, in the subset of younger patients with no fever, tachypnea, hypoxia, or abnormal breath sounds, it is not known if failure or delay of diagnosis has any impact on morbidity or mortality.

This argument cannot be made in the $1 \%$ of patients who would have had missed effusions/pulmonary edema. Two of the 3 patients with pleural effusions were admitted to the hospital and were given a new diagnosis of CHF. In review of these patients' charts, there was no single feature that suggested itself as a possible criterion to further refine decision making in ordering chest radiographs in the atraumatic chest pain patient. There was no group of historical elements nor were there exam findings in the patients presenting with chest pain that would have identified $100 \%$ of the patients with clinically significant chest radiograph findings.

These results raise the question as to whether a miss rate of $2 \%$ is acceptable and whether obtaining chest radiographs on all patients with atraumatic chest pain is an appropriate use of resources. In a population with a low incidence of disease, using criteria similar to Rothrock's could potentially reduce chest radiography by up to $65 \%$, with the attendant cost savings, reduced length of stay, and decreased radiation burden that would follow. This may be reasonable in our population, although, in a population with a higher incidence of disease, a sensitivity of $80 \%$ would be unacceptably low.

\section{Limitations}

Our study has some methodological limitations which are expected of retrospective studies. We did not differentiate the quality of chest pain within the chart review, notably because quality of chest pain is frequently not included in ED charting. We did not differentiate the length of time each patient had the chest pain, which could possibly have an association 
with pathology. This study potentially introduces spectrum bias with the sample pool neglecting rarer conditions, as well as possibly being too small to accurately represent the prevalence of disease within the community. We determined which radiograph findings were significant and valuable to our practice patterns via a priori consideration. Given that our sample size was less than 1,000 subjects, we were unable to include rarer acute findings on chest radiographs that would be significant in the population at large such as malignancy.

As noted in the methods, if a variable was not mentioned in the record, it was assessed as not being present. As with all retrospective studies involving chart review, we are unable to ensure that a variable that was not assessed or charted in the $\mathrm{ED}$ evaluation was in fact "negative" and not present in the patient.

With only 50 positive radiographs in our sample population and by using recursive partitioning to demonstrate which factors are predictive of disease, we run the risk of "overfitting" the data and will ultimately reduce the success of the rule upon prospective validation because it will not generalize well. Prospective validation of the rule in a multicenter study would have the ability to eliminate some spectrum bias as well as increasing the proportion of positive chest radiographs.

\section{Conclusion}

In our patient population, the incidence of clinically significant positive chest radiograph findings for patients presenting to the ED with nontraumatic chest pain was only $5 \%$. Of these, $80 \%$ were identified using the modified Rothrock criteria, with a negative predictive value of $98 \%$ (only $2 \%$ of patients would have had a missed pathology).

Modifying these criteria to include an older age group $(\geq 65)$ and remove history of thromboembolic disease, hemoptysis, or tuberculosis preserved the sensitivity and negative predictive value of the rule and increased its specificity. The clinical decision rule suggesting that a chest radiograph should be obtained in a patient with chest pain who is $\geq 65$ years and has a history of alcohol abuse or CHF or exam findings of temperature $>100.4^{\circ} \mathrm{F}, \mathrm{RR}>24$, or diminished breath sounds has a sensitivity of $74 \%$ (95\% CI $59-85 \%)$, a specificity of $69 \%$ (95\% CI 66-72\%), a PPV of 11 (95\% CI 8$16 \%$ ), and an NPV of $98 \%$ (95\% CI 96-99\%).

Our study suggests that it is reasonable to consider forgoing routine chest radiography in adult patients with nontraumatic chest pain that do not demonstrate any of the variables of the modified Rothrock criteria. However, emergency departments with a higher incidence of clinically significant positive chest radiograph findings may have an unacceptable miss rate. Further research with a planned prospective study on this topic will be needed to confirm our findings.

\section{Disclosure}

This paper was an award-winning poster presentation at the National Meeting of Society of Academic Emergency
Medicine, Atlanta, GA, 2013; an award-winning poster presentation at the National Meeting of American College of Emergency Physicians, Seattle, WA, 2013; an award-winning oral presentation at St. Luke's University Research Forum (first prize), Bethlehem, PA, 2013; and an award-winning poster presentation at St. Luke's University Research Forum, 8 Bethlehem, PA, 2013. Although this research was accepted for presentation at all listed meetings, which is an honor, it truly only won an award at the St. Luke's research forum, where it took home first place.

\section{Conflict of Interests}

All authors have no conflict of interests to report.

\section{Authors' Contribution}

All authors contributed substantively to the paper.

\section{References}

[1] E. W. Nawar, R. W. Niska, and J. Xu, "National Hospital Ambulatory Medical Care Survey: 2005 emergency department summary," Advance Data, no. 386, pp. 1-32, 2007.

[2] P. J. Zimetbaum and M. E. Josephson, "Use of the electrocardiogram in acute myocardial infarction," The New England Journal of Medicine, vol. 348, no. 10, pp. 933-940, 2003.

[3] A. S. Jaffe, L. Babuin, and F. S. Apple, "Biomarkers in acute cardiac disease. The present and the future," Journal of the American College of Cardiology, vol. 48, no. 1, pp. 1-11, 2006.

[4] S. G. Rothrock, S. M. Green, K. A. Costanzo, J.-M. Fanelli, E. S. Cruzen, and J. R. Pagane, "High yield criteria for obtaining nontrauma chest radiography in the adult Emergency Department population," Journal of Emergency Medicine, vol. 23, no. 2, pp. 117-124, 2002.

[5] E. P. Hess, J. J. Perry, P. Ladouceur, G. A. Wells, and I. G. Stiell, "Derivation of a clinical decision rule for chest radiography in emergency department patients with chest pain and possible acute coronary syndrome," Canadian Journal of Emergency Medicine, vol. 12, no. 2, pp. 128-134, 2010.

[6] J. K. Poku, V. R. Bellamkonda-Athmaram, M. Fernanda Bellolio, D. M. Nestler, I. G. Stiell, and E. P. Hess, "Failure of prospective validation and derivation of a refined clinical decision rule for chest radiography in emergency department patients with chest pain and possible acute coronary syndrome," Academic Emergency Medicine, vol. 19, no. 9, pp. 1004-1010, 2012.

[7] P. A. Templeton, W. A. McCallion, L. A. McKinney, and H. K. Wilson, "Chest pain in the accident and emergency department: is chest radiography worthwhile?" Archives of Emergency Medicine, vol. 8, no. 2, pp. 97-101, 1991. 


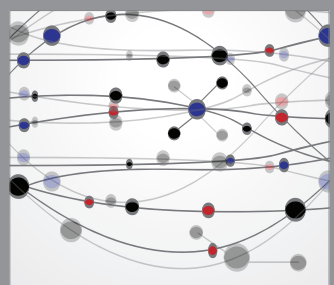

The Scientific World Journal
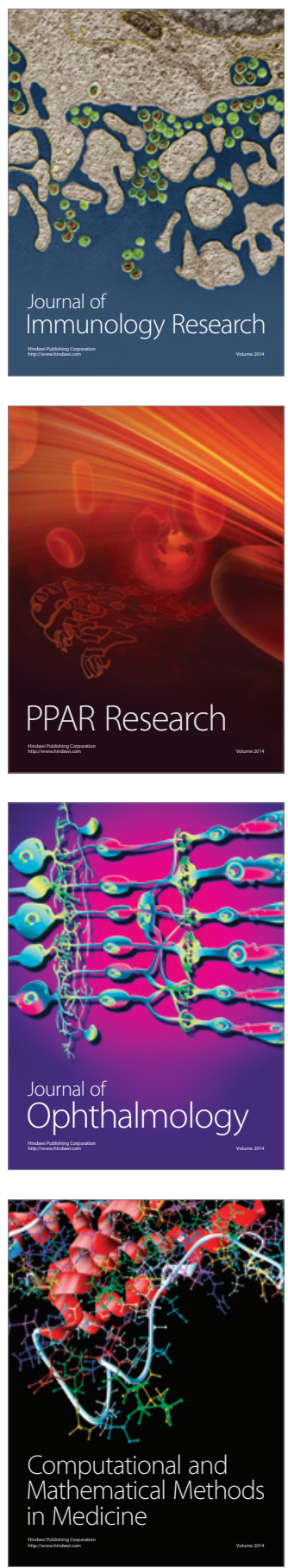

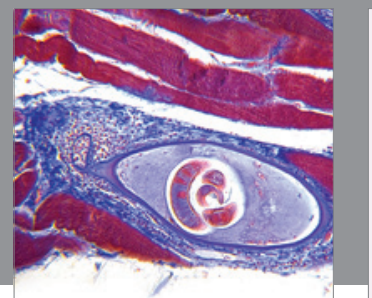

Gastroenterology

Research and Practice
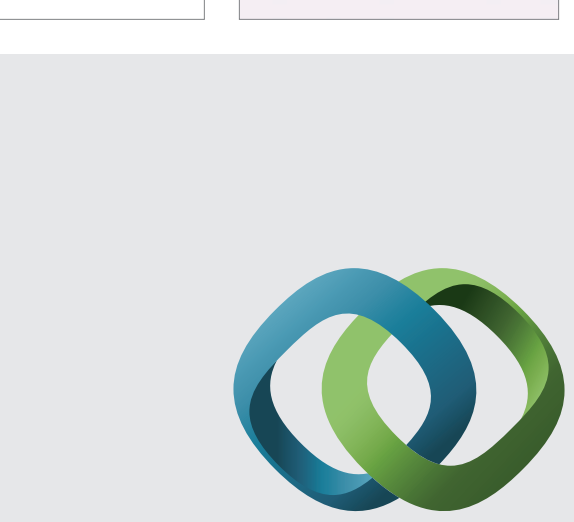

\section{Hindawi}

Submit your manuscripts at

http://www.hindawi.com
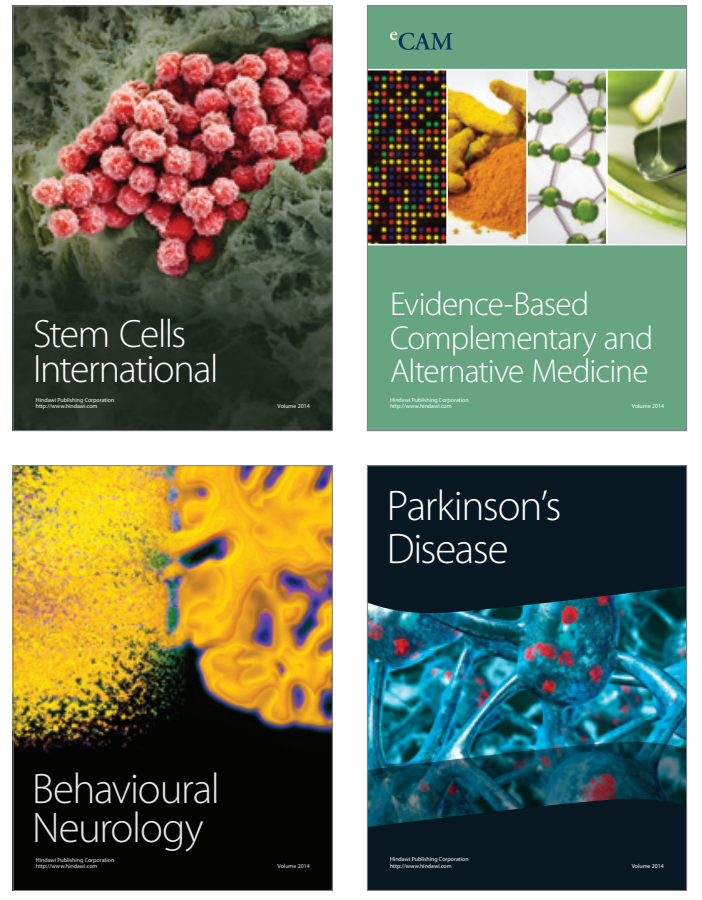
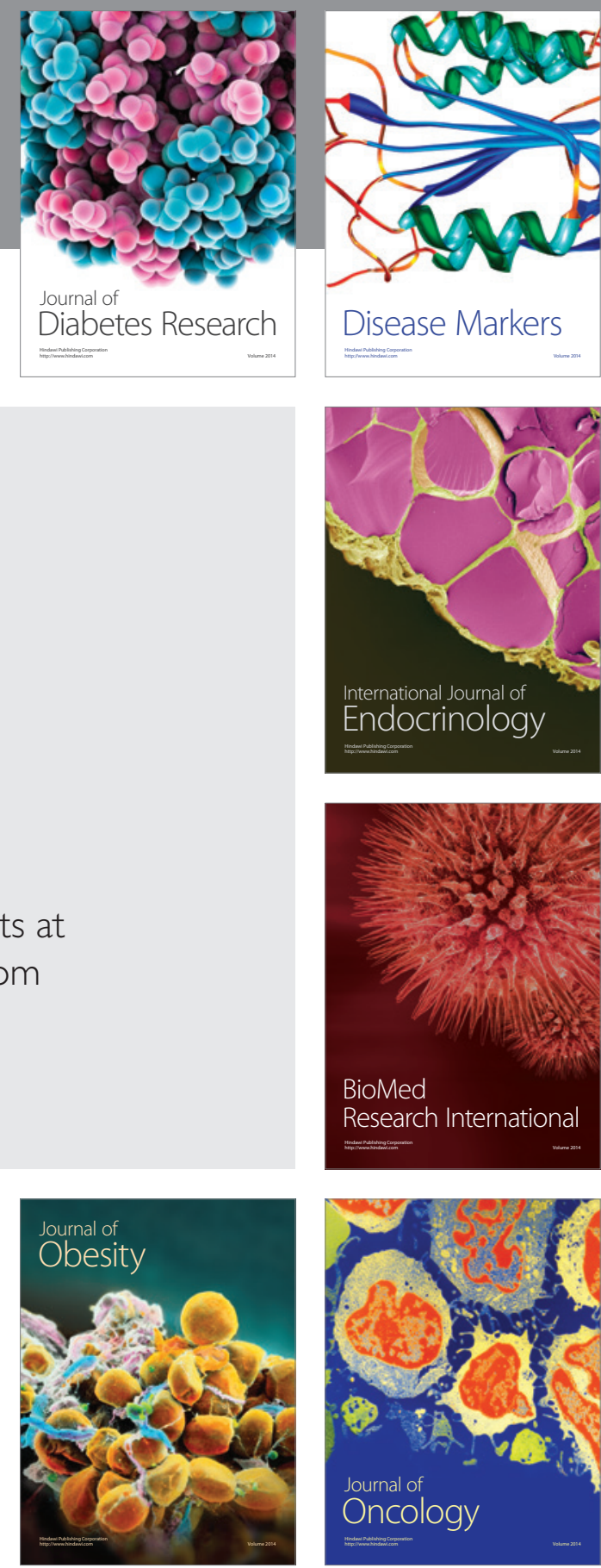

Disease Markers
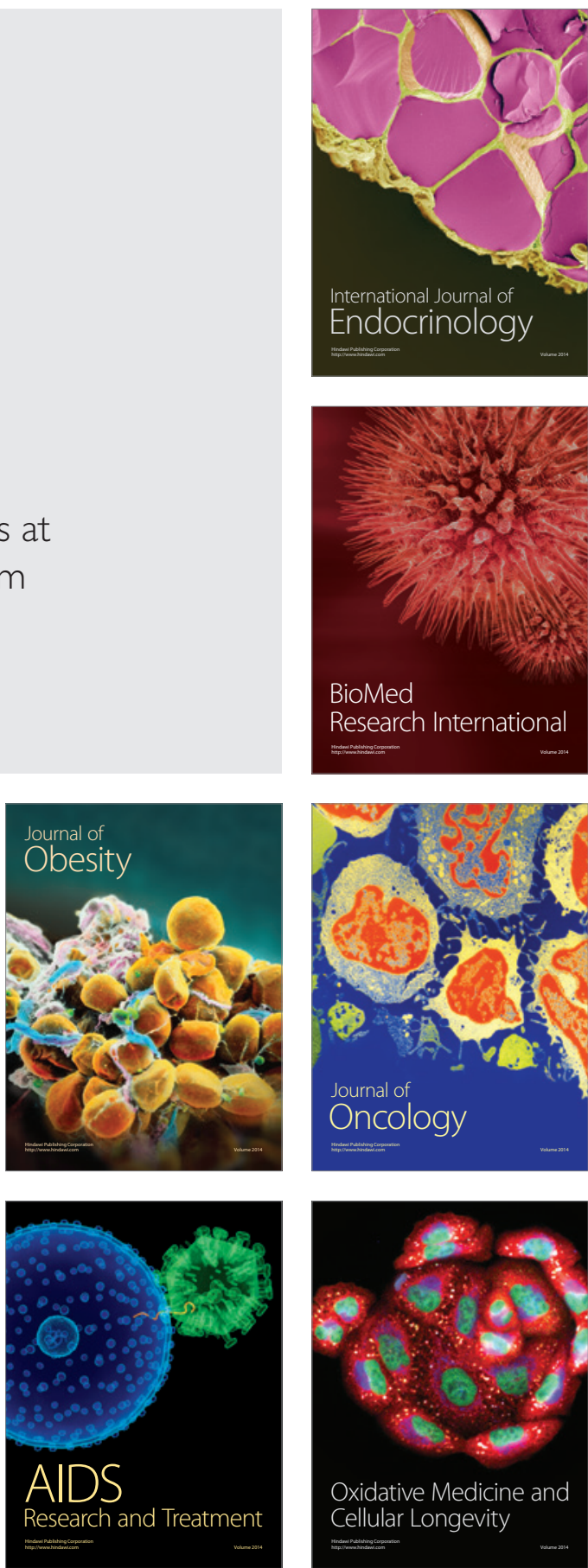